$$
\text { CONF-980696-- }
$$

Author(s):

JOSEPH T. MANG

CARY B. SKIDMORE

REX P. HJELM

PHILIP M. HOWE

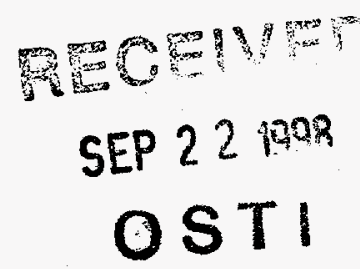

Los Alamos National Laboratory, an affirmative action/equal opportunity employer, is operated by the University of California for the

U.S. Department of Energy under contract W-7405-ENG-36. By acceptance of this article, the publisher recognizes that the U.S.

Government retains a nonexclusive, royalty-free license to publish or reproduce the published form of this contribution, or to allow

others to do so, for U.S. Government purposes. Los Alamos National Laboratory requests that the publisher identify this article as work performed under the auspices of the U.S. Department of Energy. The Los Alamos National Laboratory strongly supports academic freedom and a researcher's right to publish; as an institution, however, the Laboratory does not endorse the viewpoint of a publication or. guarantee its technical correctness. 


\section{DISCLAIMER}

This report was prepared as an account of work sponsored by an agency of the United States Government. Neither the United States Government nor any agency thereof, nor any of their employees, makes any warranty, express or implied, or assumes any legal liability or responsibility for the accuracy, completeness, or usefulness of any information, apparatus, product, or process disclosed, or represents that its use would not infringe privately owned rights. Reference herein to any specific commercial product, process, or service by trade name, trademark, manufacturer, or otherwise does not necessarily constitute or imply its endorsement, recommendation, or favoring by the United States Government or any agency thereof. The views and opinions of authors expressed herein do not necessarily state or reflect those of the United States Government or any agency thereof. 


\section{DISCLAIMER}

Portions of this document may be illegible in electronic image products. Images are produced from the best available original document. 


\title{
QUANTIFICATION OF MICROSTRUCTURAL FEATURES IN HMX USING SMALL ANGLE NEUTRON SCATTERING TECHNIQUES
}

\author{
Joseph T. Mang and Cary B. Skidmore \\ DX-2, Los Alamos National Laboratory \\ Los Alamos, NM 87545 \\ Rex P. Hjelm \\ Manuel Lujan Jr. Neutron Scattering Center, Los Alamos National Laboratory \\ Los Alamos, NM 87545 \\ Philip M. Howe \\ DX, Los Alamos National Laboratory \\ Los Alamos, NM 87545
}

\begin{abstract}
Microstructural features in raw powders of High Explosives have been qualitatively observed by many researchers, using polarized light and scanning electron microscopy. Here, we present a method for non-destructive quantification of volume fraction and structure of intragranular cracks and crystallization voids in a bulk sample (100-300 mg). By employing Small Angle Neutron Scattering (SANS) in conjunction with the method of contrast variation, we can effectively highlight different structural features of a complex system. The technique of contrast variation relies on immersing the sample in a uniform fluid of known neutron scattering length density. By selectively varying the scattering length density of the immersion fluid, scattering contributions from internal and external structures can be separated. This approach is analogous to varying the index of refraction for immersion oil relative to a sample in polarized light microscopy.

SANS experiments on HMX were conducted using loose powders (261 and 10 micron mean particle diameters) and pellets made by uniaxial consolidation (without binder) to 7 and 10 volume percent porosity respectively. Detailed modeling of the SANS data indicate significant alteration of the intragranular void/crack/pore structure, with pressing, of the HMX powders.
\end{abstract}

\section{Introduction}

High explosive (HE) materials are frequently composites, consisting of a crystalline high explosive and a polymeric binder. These materials possess both naturally occurring and processrelated defects (cracks, voids, etc.) which can affect their response to certain stimuli. Under conditions of impact or shock for example, locally heated regions, hot spots, can occur. The creation of hot spots is thought to occur by conversion of mechanical work into thermal energy through processes such as viscous void collapse ${ }^{1}$. The critical hot spot temperature is known to 
be dependent upon both the size and shape of the defect, so shifts in the pore size distribution in an explosive can markedly change the explosive response to shock and impact stimuli ${ }^{1}$. Larger pores ( $>1 \mu \mathrm{m}$ diameter) have lower critical temperatures and are generally thought to be of more consequence in generating hot spots. However, recent molecular dynamics simulations ${ }^{2}$ have shown that nanometer sized defects interact strongly with shock waves and thus have profound effects on the shock to detonation transition. In order to quantitatively describe an explosive's response from the lowest ignition thresholds to the development of full detonations and explosions, accurate measurements of a number of structural parameters of the $\mathrm{HE}$ composite, particularly porosity and pore size distributions are required.

Experimental efforts at Los Alamos National Laboratory are aimed at providing detailed structural information of high explosives over lengthscales from angstroms to microns. Such efforts require the use of a variety of techniques, including optical microscopy, BET and SEM. However, direct, quantitative determination of structural parameters is needed for an accurate description of HE materials. Standard metallographic techniques, such as cutting and polishing samples are adequate for measuring particle size distributions, but are inadequate for measuring pore size distributions because the softness of the materials may cause the surface pores to be filled in polishing. Techniques such as BET adsorption or mercury porosimetry measure only open pores, which represent only a fraction of the total porosity in design explosives. As part of this ongoing effort, we have performed small angle neutron scattering (SANS) measurements in conjunction with the method of contrast variation (to be discussed later in the paper) to measure the shape and internal structure of neat HMX powders. In comparison to other techniques, SANS has a distinct advantage in that it is noninvasive. No special treatment (polishing, grinding, etc.) of samples is necessary for study. A sample can then be examined in its pristine state, exposed to some external insult and then reexamined without further altering the state of the material. In this way, in situ measurements of the effects of external stimuli on structural parameters can be made, allowing for more direct correlation between cause and effect. SANS can provide detailed structural information over length scales between 10 and $1000 \AA$. As the scattered neutron intensity is proportional to the number and squared volume of the particles present, SANS can provide quantitative information about pore size distributions. Over length scales larger than $0.1 \mu \mathrm{m}$, SANS can provide in situ measurements of surface area. 


\section{Small Angle Neutron Scattering}

In a SANS experiment, a collimated beam of neutrons impinges upon a sample, which is characterized by a scattering length density $\rho(\mathbf{r})$. At any point within the sample, the scattering length density is equal to the sum of the atomic neutron scattering amplitudes (scattering lengths) in a small volume around that point, divided by the volume ${ }^{3}$ :

$$
\rho=\frac{\sum_{i} n_{i} b_{i}}{V_{m}}
$$

Here, $b_{i}$ is the atomic neutron scattering length, $n_{i}$ the number of a given atom in a molecule and $V_{m}$ is the molecular volume. A fraction of the incident neutrons will be scattered through an angle, $2 \theta$, from fluctuations in the scattering length density. $\rho(\mathbf{r})$ then reflects microscale structure in the sample in both density and chemical composition.

The scattered intensity, $I(Q)$, is measured as the absolute differential cross section per unit scattering mass $\left(\mathrm{cm}^{2} \mathrm{~g}^{-1}\right)$ as a function of the magnitude of the scattering vector, $\mathrm{Q}$. For elastic scattering events, $Q=(4 \pi / \lambda) \sin \theta$, where $\lambda$ is the wavelength of the incident neutron and $\theta$ is half of the scattering angle. $I(Q)$ is related to $\rho(\mathbf{r})$ by a squared Fourier transform ${ }^{3}$ :

$$
I(Q)=K\left\langle\left|\int \rho(\mathbf{r}) \exp (-i \mathbf{r} \cdot \mathbf{Q}) d r\right|^{2}\right\rangle=K P(\mathbf{Q}),
$$

where, the angle brackets indicate a spherical average over all possible particle orientations and $P(Q)$ is the normalized particle form factor, which contains the particle shape information. The constant, $\mathrm{K}=\mathrm{NV}^{2} \Delta \rho^{2} \mathrm{M}$, where $\mathrm{N}$ is the number of particles, $\mathrm{V}$ is the volume of a particle, $\mathrm{M}$ is the scattering mass and $\Delta \rho=\bar{\rho}-\rho_{s}$ is the contrast between the average scattering length density of the particle, $\bar{\rho}$ and that of the surrounding media, $\rho_{s}$.

Eq. 2 describes the observed scattering over all possible length scales. Important approximations to this expression exist which are valid over much more limited ranges. For example, in the range such that $Q R<1$, where $R$ is the average radius of the particles under study, the observed scattering can be described by the Guinier approximation ${ }^{3}$ :

$$
I(Q)=K e^{-Q^{2} R_{s}^{2} / 3} .
$$

In Eq. 3, $R_{g}$ is the particle radius of gyration and for spheres, $R_{g}{ }^{2}=3 / 5 R^{2}$. Over length scales 
such that $\mathrm{QR} \gg>1$, the scattering can be described as a negative power-law ${ }^{4}, I(Q)=I_{o} Q^{-\alpha}$, where $\alpha$ and $I_{o}$ are constants. For rough interfaces, $3 \leq \alpha<4$. For the special case of smooth interfaces, $\alpha=4$ and the scattering is termed Porod scattering. Under these circumstances,

$$
I(Q)=2 \pi S \Delta \rho^{2} Q^{-4}
$$

where $S$ is the interfacial area per gram of material ${ }^{3}$.

\section{Scattering From Simple Shapes}

Well-defined particles give rise to distinctive se pring patterns as a function of $\mathrm{Q}$. For example, in the case of a spherical particle of radius $\mathrm{R}$; the particle form factor has the form ${ }^{3}$ :

$$
P(Q)=\left|\frac{3 j_{1}(Q R)}{Q R}\right|^{2},
$$

where $j_{1}$ is the first order spherical Bessel function. The anticipated scattering for an isotropic solution of monodisperse spherical particles is shown in Fig. 1. As seen in the figure, in the low$\mathrm{Q}$ region, the scattering curve follows the Guinier approximation (Eq. 3). This regime is followed by successive maxima which fall-off in intensity as $\mathrm{Q}^{-4}$. The positions of the maxima are indicative of the particle radius. In general, most systems contain some level of size polydispersity, the effects of which can be taken into account by performing the appropriate population average of the form factor. The scattered intensity then has the form:

$$
I(Q)=\frac{\Delta \rho^{2}}{M} \int_{0}^{\infty} N(R) V(R)^{2} P(\mathbf{Q}, R) d R
$$

As seen by the solid line in Fig. 1, where we have assumed a Gaussian distribution of spherical particles, the effect of polydispersity (even at low-levels) is to wash-out the maxima, resulting in a loss of information.

\section{Method of Contrast Variation}

In more complex systems, such as composite materials ${ }^{5,6}$, determination of the structureproperty relationships is more difficult. This is due to the fact that there are now multiple contributions to the scattering signal. For an HE system, these contributions arise from the 


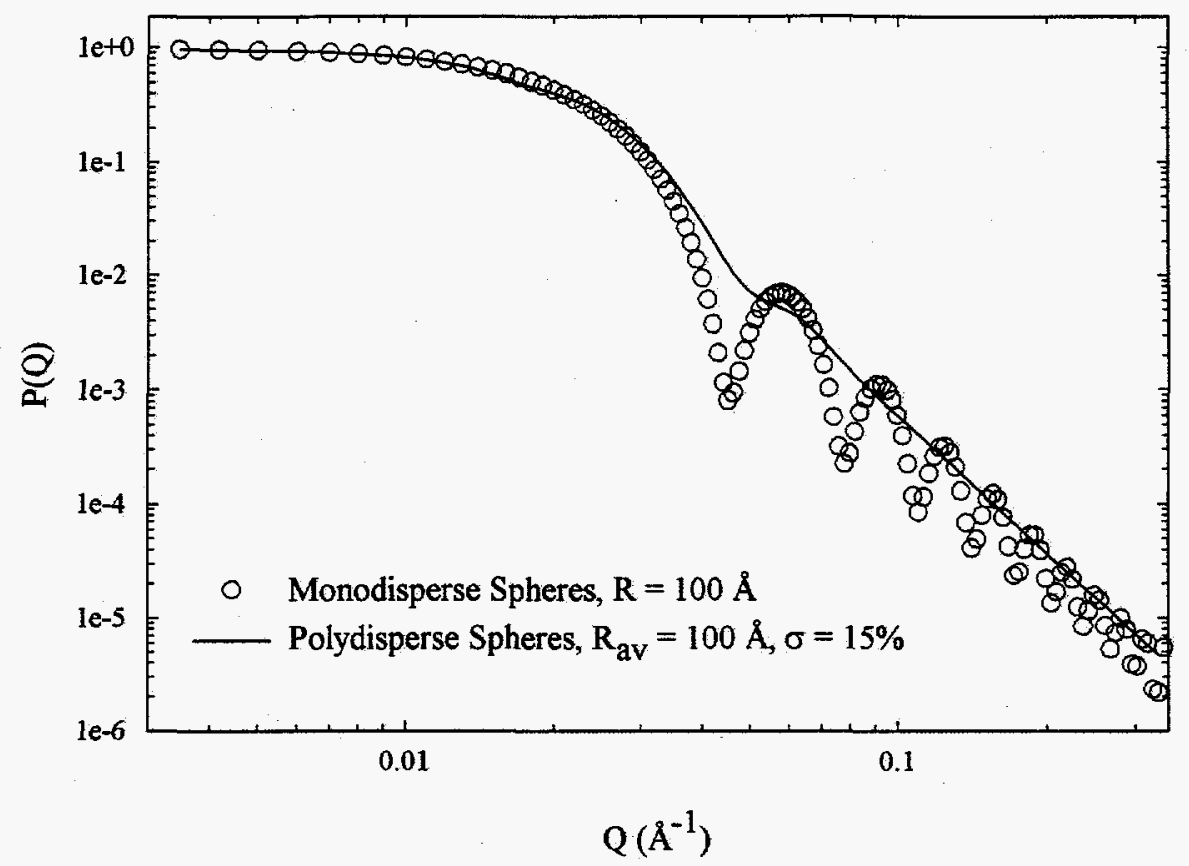

Figure 1 : Modeled SANS scattering for monodisperse spheres $(R=100 \AA)$ and a Gaussian distribution of spheres $\left(R_{\mathrm{av}}=100, \sigma=15 \%\right)$.

polymer matrix, the filler (HE material) and the matrix-filler interface as well as from defects, such as (internal and external) voids and cracks. The first step in sorting-out the sources of the observed scattering is to study the isolated components. But, even the investigation of these simplified systems, because of the possibility of internal structure, requires an additional technique, the method of contrast variation, to separate the scattering arising from the shape from that of the internal structure. The method takes advantage of the large differences in neutron scattering lengths among isotopes of hydrogen, and the consequential difference in scattering length density between deuterated and non-deuterated materials. Thus, making a fluid mixture of deuterated and non-dueterated species (cyclohexane and deuterocyclohexane in this work) in different proportions, $\Delta \rho$ in Eq. 2 can be varied continuously and different structural features enhanced or suppressed (Fig. 2).

For the purpose of contrast variation we can think of the sample as a collection of particles suspended in a uniform fluid, then the structure of the particles can be modeled as ${ }^{3,5}$ :

$$
\rho(\mathbf{r})=\rho_{s}+\left(\bar{\rho}-\rho_{s}\right) \Omega(\mathbf{r})+\zeta(\mathbf{r})
$$



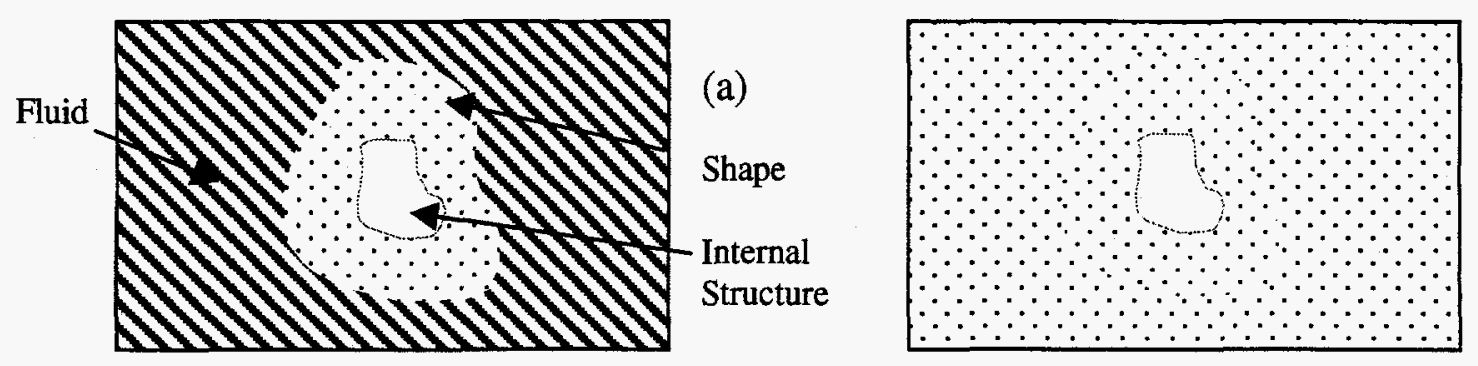

Figure $2:$ By varying the scattering length density of the surrounding media, different structural features can be highlighted. At infinite contrast (a) we see the particle shape and at the contrast match point (b), we see the internal structure.

where, $\rho_{s}$ is the scattering length density of the fluid and $\bar{\rho}$ is the average value of $\rho(\mathbf{r})$ over the entire sample volume, $V$, given by the expression,

$$
\bar{\rho}=\frac{\sum_{i} n_{i} b_{i}}{V} .
$$

The scattering contrast is defined as $\Delta \rho=\bar{\rho}-\rho_{s}$, as before. Now, two important, limiting cases need to be considered. At very high contrast ( $\sim$ infinite), the internal structure of an object makes no significant contribution to the overall scattering. Under these circumstances, we measure the shape of the particles, represented by $\Omega(\mathbf{r})$ in Eq. 7 (Fig. 2a). This is the shape of the fluid excluding parts of the object. At the other extreme, $\Delta \rho \rightarrow 0$ (the contrast match point), only fluctuations from the internal structure $(\zeta(\mathbf{r}))$, contribute to the scattering (Fig. 2b). At any given contrast, the observed scattering intensity is proportional to the square of the Fourier transform of Eq. 7 and can be expressed as a quadratic in $\Delta \rho$ :

$$
I(\Delta \rho, Q)=\Delta \rho^{2} I_{\Omega}(Q)+\Delta \rho I_{\Omega \zeta}(Q)+I_{\zeta}(Q) .
$$

The coefficients, $I_{\Omega}(Q), I_{\Omega \zeta}(Q)$ and $I_{\zeta}(Q)$ represent the scattering from the shape of the solvent excluding regions, the scattering due to correlations between the shape and internal structure and the scattering from the internal structure, respectively. In an experiment, data is collected as a function of $\Delta \rho$ and $\mathrm{Q}$. The resulting curves are then fit to Eq. 9 at each value of $\mathrm{Q}$ and the three scattering functions determined. A minimum of three different contrasts must be studied to make an unambiguous determination of the individual functions. Measurements are typically performed at, above and below the contrast match point.

It should be noted that in arriving at Eq. 9, we have assumed a chemically homogeneous 
system. If chemical inhomogeneity exists, the expressions for the individual scattering functions become more complex ${ }^{6}$. To the extent that the system can be approximated as chemically homogenous, Eq. 9 is valid. As will be shown later, the validity of this assumption can be tested in the normal course of data analysis.

\section{Experimental}

Small angle neutron scattering measurements were performed on the Low-Q Diffractometer (LQD) at the Los Alamos Neutron Science Center (LANSCE). Data were reduced by conventional methods ${ }^{7}$ and are reported as differential scattering cross section per unit mass, $\mathrm{I}(\mathrm{Q})\left(\mathrm{cm}^{2} \mathrm{~g}^{-1}\right)$, as a function of the scattering vector, $\mathrm{Q}$.

Experiments were performed on powders of both coarse and fine $\mathrm{HMX}$, having mean particle diameters of 261 and $10 \mu \mathrm{m}$, respectively. In addition, pressed pellets, made by uniaxial consolidation of the neat material to 7 (coarse) and 10 (fine) volume percent porosity were investigated in order to determine the effect of pressing on the microstructure. The scattering measurements were performed in conjunction with the method of contrast variation, as described previously. To this end, all samples were mixed with cyclohexane and placed in rectangular quartz cells having a $2 \mathrm{~mm}$ pathlength. The choice of a dispersion fluid was based upon the wide range of contrasts that can be reached with cyclohexane/deuterated cyclohexane mixtures and that HMX is insoluble in cyclohexane. The ratio of deuterated/non-deuterated cyclohexane was varied in order change the total scattering contrast of a sample. Five to six different contrasts were measured for each sample. The data from the individual runs were fit according to Eq. 9. The results obtained for the shape and internal structure functions are discussed below.

\section{Results and Discussion}

SANS lineshapes for the loose coarse (HMX-C), pressed coarse (C93), loose fine (HMXF) and pressed fine (F90) at different contrasts are shown in Fig. 3. As the amount of deuterated solvent is varied (contrast is varied), a change in both intensity and shape of the curves is seen. 


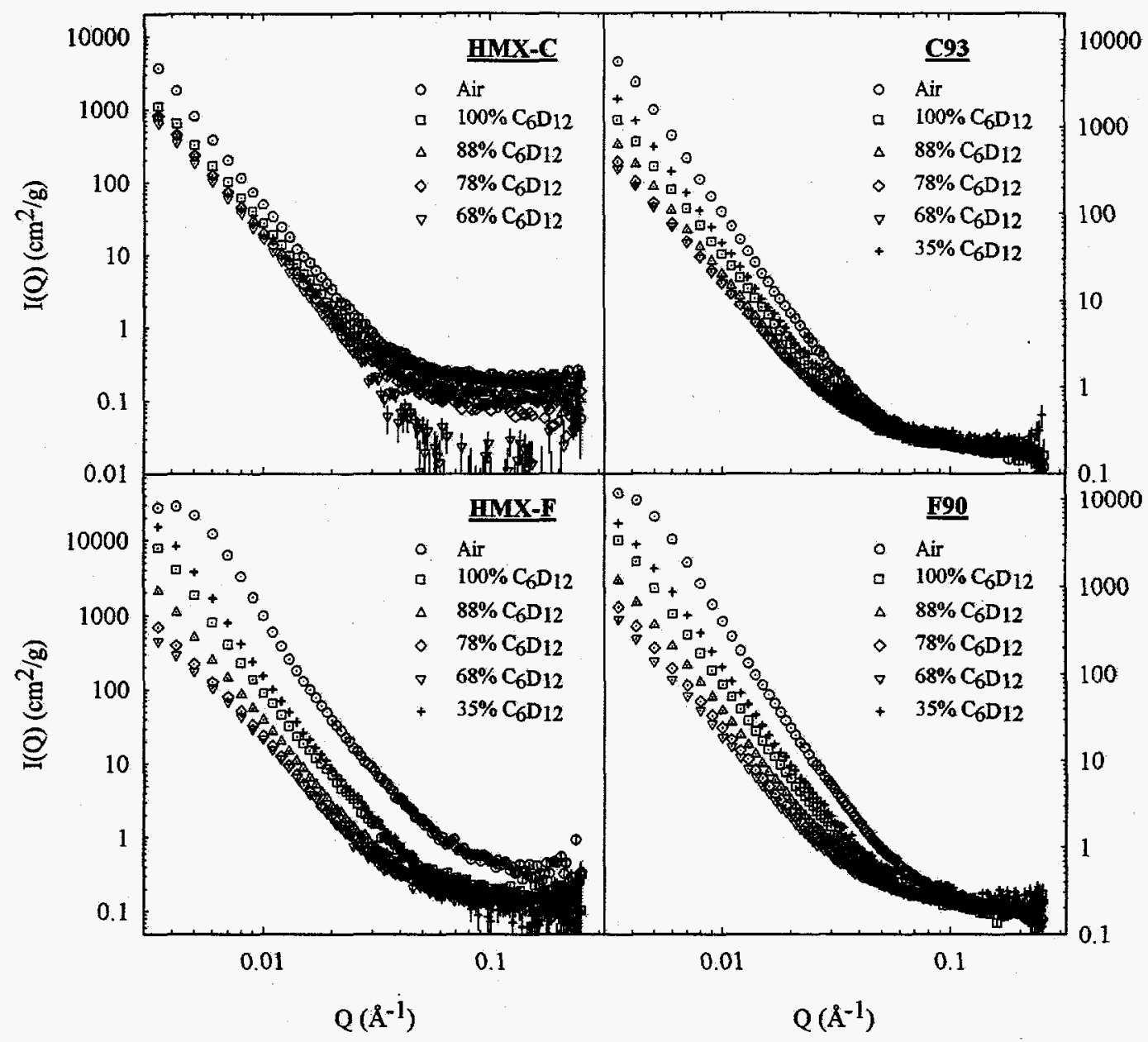

Figure 3 : SANS scattering curves as a function of contrast for HMX powders. Curves change in intensity and shape as contrast is varied because of internal structure.

This initial observation is a good qualitative indication that the HMX crystals possess internal structure over the length scales probed $(10-1000 \AA)$ and that the SANS technique is sensitive to this structure.

Analysis of the lineshapes according to Eq. 9 is displayed in Fig. 4 in the form of contrast maps, which show clear minima as $Q \rightarrow 0$. The zero of contrast along the vertical axes was calculated using the scattering length density predicted by the chemical composition of HMX $\left(\rho=4.5 \times 10^{10} \mathrm{~cm}^{-2}\right)$. The positions of the minima indicate the average scattering length density $(\bar{\rho})$ of the HMX material. As can be seen in the figure, in all cases the minima are displaced from zero indicating that $\bar{\rho}$ is different from that predicted by the chemical 


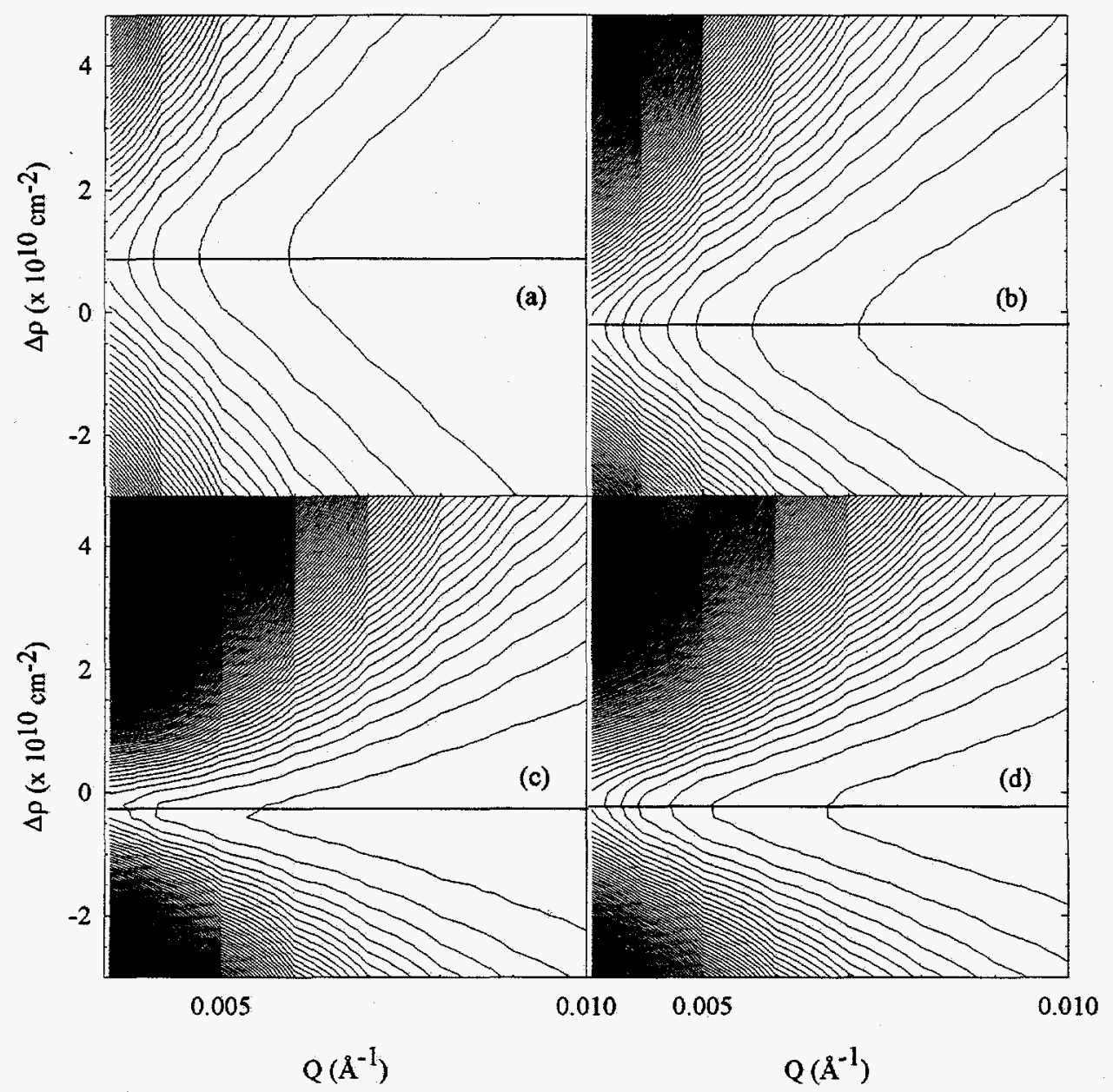

Figure 4 : Contrast maps for HMX powders. (a) HMX-C, (b) C93, (c) HMX-F, (d) F90. Position of contour minima determines $\bar{\rho}$.

composition. Except for the case of HMX-C, the minima appear at negative values of $\Delta \rho$, indicating that $\bar{\rho}$ is less than $\rho$. This is what would be expected if the differences in scattering length density are due to the presence of voids, internal to the HMX crystals. With the use of Eqs. 1 and 8, we can derive an expression for the void fraction, $\phi_{\nu}$, in terms of $\rho$ and $\bar{\rho}$ :

$$
\phi_{v}=1-\frac{\bar{\rho}}{\rho} \text {. }
$$

Values of $\phi_{v}$ calculated from Eq. 10 and the $\bar{\rho}$ determined from the contrast maps are summarized in Table I. In comparing the HMX-F and F90 samples, we see that there is a slight decrease in the void fraction with pressing. This suggests that the internal voids are collapsing 
Table I : Summary of internal void fraction and mean internal pore size for HMX powders.

\begin{tabular}{cccc}
\hline \hline Sample & Internal Void fraction, $\phi$, & Mean Pore Diameter $(\AA)$ & Diameter RMS $(\AA)$ \\
\hline HMX-C & --- & 560 & 320 \\
HMX-F & 0.08 & 112 & 104 \\
C93 & 0.04 & 98 & 46 \\
F90 & 0.05 & 34 & 10 \\
\hline \hline
\end{tabular}

under pressure. We were unable to make a clear determination of $\phi_{\nu}$ for the HMX-C sample so we cannot make a direct comparison of the coarse material. However, the value measured for the C93 is very near that of F90, which is consistent with the idea of collapsing voids.

While the contours of Fig. 4 have well-defined minima, a small Q-dependence to the minima positions was found, suggesting the possible influence of chemical inhomogeneity on the experimental results. Chemical analysis of the HMX showed only a small amount $(<1 \%)$ of RDX impurity which will have negligible effect on the determination of $\bar{\rho}$. If large chemical inhomogeneities existed in the system, the contours in Fig. 4 would appear to be flattened-out. So the appearance of sharp minima in Fig. 4 is a good indication of chemical homogeneity. The basic scattering functions were calculated for values of $\rho$ ranging from $4.5-4.9 \times 10^{10} \mathrm{~cm}^{-2}$ (as determined from Fig. 4) to determine the influence of $\rho$ on the analysis. Over the range of scattering length densities used, no significant changes in either $I_{\Omega}$ or $I_{\zeta}$ were observed.

The basic scattering functions derived from the data are shown in Figs. 5-6. Fig. 5a shows the shape functions for all four samples which display power-law scattering with an exponent, $\alpha$ $=4$. Analysis of the shape functions was carried-out according to Porod's law (Eq. 4) and the total surface area per gram determined. The results are summarized in Fig. $5 b$. As seen in the figure, the HMX-F sample has a much higher surface area than the HMX-C sample. This is expected as the fine material is made by grinding the coarse HMX into smaller particles. Upon pressing, an increase of surface area was found in the case of the coarse material, suggesting that pressing induces surface cracks and breaks-up crystallites. However, in the case of the fine material, a slight loss of surface area upon pressing is seen. This behavior can be understood by considering that when a material is pressed, several things happen which contribute to the surface area. First, there is a gain in surface area when crystallites are broken-up or when surface cracks are induced. But, at the same time, there is a loss in surface area to the collapse of existing surface cracks and to increased contact of individual crystallites. So, for the coarse 
material, the dominant effect is the breaking and cracking of crystallites, resulting in a net gain in surface area, whereas for the fine HMX, the net loss in surface area suggests that the collapsing of cracks and pressing of crystallites together is the dominant behavior. These results differ from those obtained by BET measurements. For the coarse HMX, the SANS results show the same trend of increasing surface area with pressing as BET, but differ in the absolute values measured. However, for the fine HMX, the two techniques differ in both magnitude and trend. The differences between the two techniques may result from the different length scales probed by the individual techniques and are currently under investigation.

The internal structure functions for the coarse and fine HMX samples are shown in Fig. 6. Significant, qualitative changes in the lineshapes are visible upon pressing, indicating that pressing induces structural changes. In order to quantify these changes, the data were fit (solid lines in the figure) to a general model consisting of polydisperse spherical particles (Eq. 6). $N(R)$ in Eq. 6 was assumed to have a Gaussian shape. From the analysis, a mean size for the internal voids (or pores) was determined. The results are summarized in Table I, where we see that the coarse material is characterized by a larger mean pore size than the fine as would be expected. In both cases, there is a shift to a smaller pore size with pressing with smaller root mean deviation
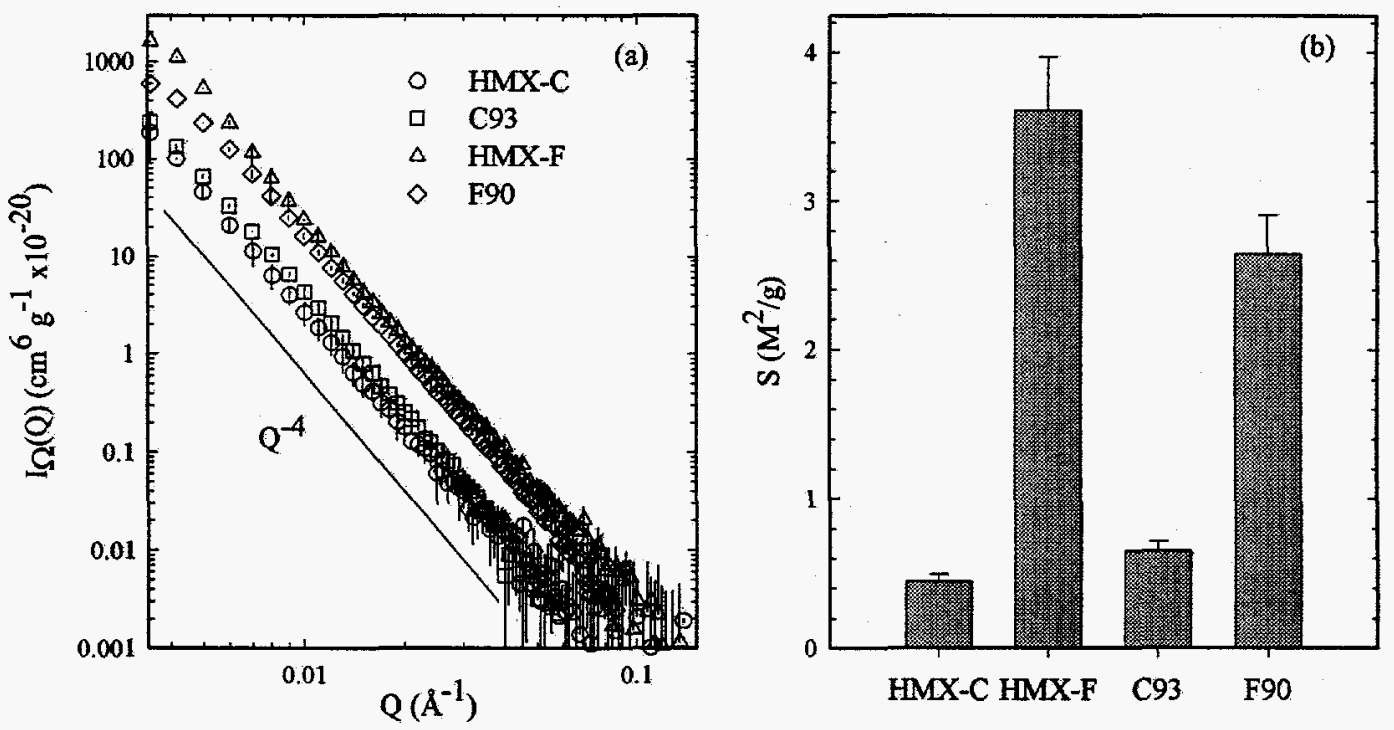

Figure 5 : (a) Shape functions determined by contrast variation analysis. (b) Surface area per gram derived from Porod analysis of shape functions. 


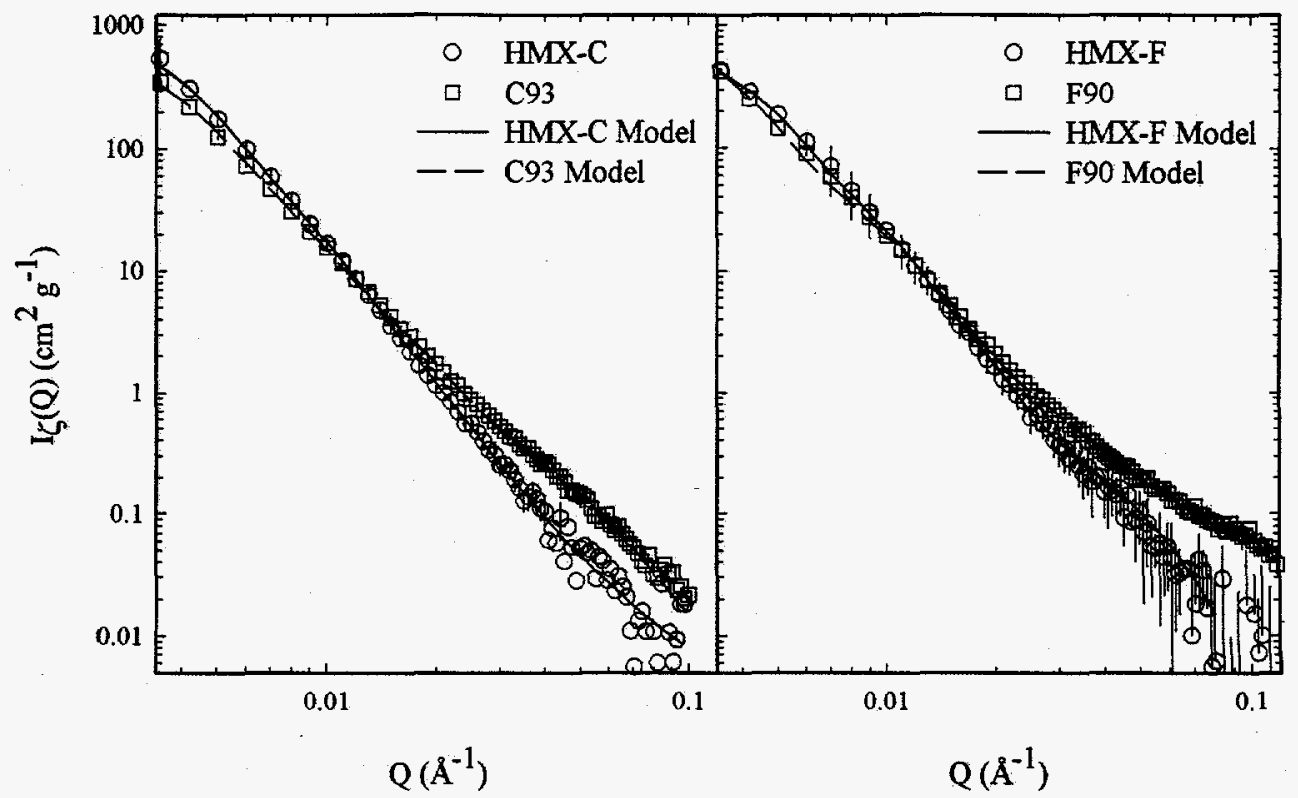

Figure 6 : Internal structure functions. Changes in measured curves indicate pore collapse upon pressing. Solid and dashed lines are fits to a model of polydisperse spheres.

(RMS) about the means. Such a shift can be caused by the collapse of larger pores and/or the induction of elongated structures (internal cracks) and is consistent with the void fraction calculations.

\section{Summary and Conclusion}

We have used small angle neutron scattering to study the microstructure of a high explosive material. The changes observed in the measured lineshapes as a function of contrast are indicative of structure, internal to the HMX material. With the use of the contrast variation technique, we were able to separate the scattering signals arising from this internal structure and that arising from the shape of the HMX crystals. Analysis of contrast maps enabled a determination of the average scattering length density, $\bar{\rho}$, and hence the void fraction. $\phi_{v}$ was found to decrease with pressing and sample preparation (grinding), suggesting a collapse of internal voids. The shape functions for all four samples studied display Porod scattering, indicating that the lower bound for particle size is $>0.1 \mu \mathrm{m}$. Through analysis of the shape 
functions we were able to measure systematic changes in the surface area as a function of pressing. By modeling the internal structure functions as a system of polydisperse spherical particles, a mean internal pore size for the different materials and an RMS of the distributions were extracted. A downward shift of the mean pore size was seen, dependent upon pressing, indicating a collapse of larger voids and/or the development of more elongated structures (cracks).

Our results demonstrate that small angle neutron scattering is a powerful tool for use in the characterization and surveillance of high explosive materials. With SANS, we can obtain in situ measurements of surface area and quantitative measurements of internal void populations as well as changes in these quantities dependent upon external insult. These preliminary results indicate that there is significant structure in HMX in the range of $10-1000 \AA$, which may influence the shock to detonation transition ${ }^{2}$. In the future we plan to study the more complex PBX9501 (95 wt.\% HMX, coarse:fine - 3:1) system in order to determine the influence of the binding material on changes in the particle structure.

This work was supported in part by the U.S. Department of Energy under contract number W7405-ENG-36 with the University of California.

\section{References}

1. Tarver, C., Chidester, S. and Nichols, A., J. Phys. Chem., 100, 5794 (1996).

2. White, C., Barrett, J., Mintmire, J., Elert, M. and Robertson, D., Decomposition, Combustion and detonation Chemistry of Energetic Materials (Materials Research Society, Pittsburgh, PA, Vol. 418, 1996).

3. Glatter, O. and Kratky, O., Small Angle X-ray Scattering (Academic Press, London, 1982).

4. Schaefer, D. and Keefer, K., Phys. Rev. Letts., 56, 2199 (1986).

5. Hjelm, R., Wampler, W. Seeger, P. and Gerspacher, M., J. Mater. Res., 9, 3210 (1994).

6. Hjelm, R., Mang, J., Skidmore, C. and Gerspacher, M., Proceedings of the Materials Research Using Cold Neutrons at Pulsed Sources Conference, World Scientific Publishing (in press).

7. Seeger, P. and Hjelm, R., J. Appl. Cryst., 24, 467 (1991). 